\title{
A Versatile Workbench Simulator: Five-phase Inverter and PMa-SynRM performance evaluation
}

\author{
Fernando Acosta-Cambranis \\ Deparment of Electronic Engineering \\ Universitat Politècnica de Catalunya \\ Terrassa, Spain \\ fernando.geovany.acosta.cambra@upc.edu
}

Tomasz Michalski

Deparment of Electronic Engineering

Universitat Politècnica de Catalunya

Terrassa, Spain

tomasz.michalski@upc.edu

\author{
Jordi Zaragoza \\ Deparment of Electronic Engineering \\ Universitat Politècnica de Catalunya \\ Terrassa, Spain \\ jordi.zaragoza-bertomeu@upc.edu \\ Viator Pou-Muñoz \\ Deparment of Electronic Engineering \\ Universitat Politècnica de Catalunya \\ Terrassa, Spain \\ viator.pou@upc.edu
}

\author{
Luis Romeral \\ Deparment of Electronic Engineering \\ Universitat Politècnica de Catalunya \\ Terrassa, Spain \\ luis.romeral@upc.edu
}

\begin{abstract}
This paper presents the design and structure of a versatile workbench simulator for evaluating the performance of a five-phase inverter and Permanent Magnet assisted Synchronous Reluctance Motor (PMa-SynRM). The simulator allows for adding variations to the modulation techniques, changing the inverter structure's semiconductor device, and calculating the inverter's power losses. It can also facilitate observing the current, voltage, and the joint temperature of the semiconductors devices. Furthermore, we can obtain a perform that is close to an actual PMa-SynRM, depending on the desired conditions of speed and torque. The workbench simulator was developed by combining three software: Matlab/Simulink, PLECS and Altair Flux.
\end{abstract}

Keywords-PMa-SynRM, five-phase inverter, simulator, power losses, Total Harmonic Distortion.

\section{INTRODUCTION}

Simulations play an important role in analyzing the behavior of an electro-mechanical system. Because systems are becoming increasingly complex in structure and operation, computer systems help analyze them before they are built, thus increasing our understanding of it. A good simulation platform (i.e. simulator) for designing, analyzing and operating a system serves as an important tool for research. Reliable simulators help reduce the time and cost of development in electronic systems. What is more, they guarantee that system performance will comply with specifications and anticipate variations while operating under specific conditions[1]-[6]. Simulators can be used for different purposes. Some applications are for academic use and others for research and prototyping, among others.

Multi-phase machines can be used in many applications such as power traction, energy generation, and industrial applications among others. Some benefits of these machines are better fault tolerance, improved torque per ampere, more degrees of freedom, higher reliability, and improved distribution of power between the inverter phase legs, among others[7]-[9].

Multi-phase machines are commonly fed by two-level inverters. Modulation techniques must be applied to the inverter in order to feed the multi-phase machines and obtain the best performance. Carrier-based PWM (CBPWM) and Space Vector PWM (SVPWM) are the most commonly used modulation techniques for these applications[10]-[13]. Each of the modulation techniques can provide different performance to our system in terms of, for example, distributing and reducing power losses, reducing of the common-mode voltage (CMV), harmonic content, current, and voltage waveform.

A variety of semiconductors provide options for choosing the best device that meets the application's requirements. The current market options among semiconductor devices are traditional Silicon $(\mathrm{Si})$, wide bandgap Silicon Carbide ( $\mathrm{SiC}$ ) and Gallium Nitride $(\mathrm{GaN})$. An analysis of these devices is developed in [14].

This simulator is based on three different software: Simulink/MATLAB, PLECS blockset for Simulink/MATLAB and Altair Flux. Simulink implements the control and modulation techniques for a five-phase voltage source inverter (VSI), which is developed in PLECS. PLECS can reproduce behavior that is close that of real semiconductor devices, such as simulating switching and conduction losses, devices voltage and currents, gate signals, joint temperature, and others. The electric motor is designed in Altair Flux, which allows for calculating the electrical and mechanic behavior of the motor in order to approach the real behavior of the machine. Fig. 1 shows the structure of the simulator.

The Simulink/MATLAB software allows a flexible and friendly way to realize control systems. A block diagram is used to design the control algorithms and run closed-loop simulations. As it is commonly used, Simulink/MATLAB has the added advantage of being able to connect and synchronize with a wide range of software.

PLECS provides an intuitive and efficient environment for modeling and simulating power electronic circuits with active, passive and electromechanical components. It represents the active components by means of a simplified behavioral semiconductor model. PLECS can calculate the switching and conduction losses with precision with manufacturer-provided multi-dimensional lookup tables.

It also provides a large library of passive components, among these are non-linear and time variant resistors, inductors and capacitors, this allows a realistic representation of saturing components. PLECS integrates easily with Simulink/MATLAB.

Altair Flux is finite element analysis (FEA) software, that provides fast and accurate results. FEA software uses mesh 


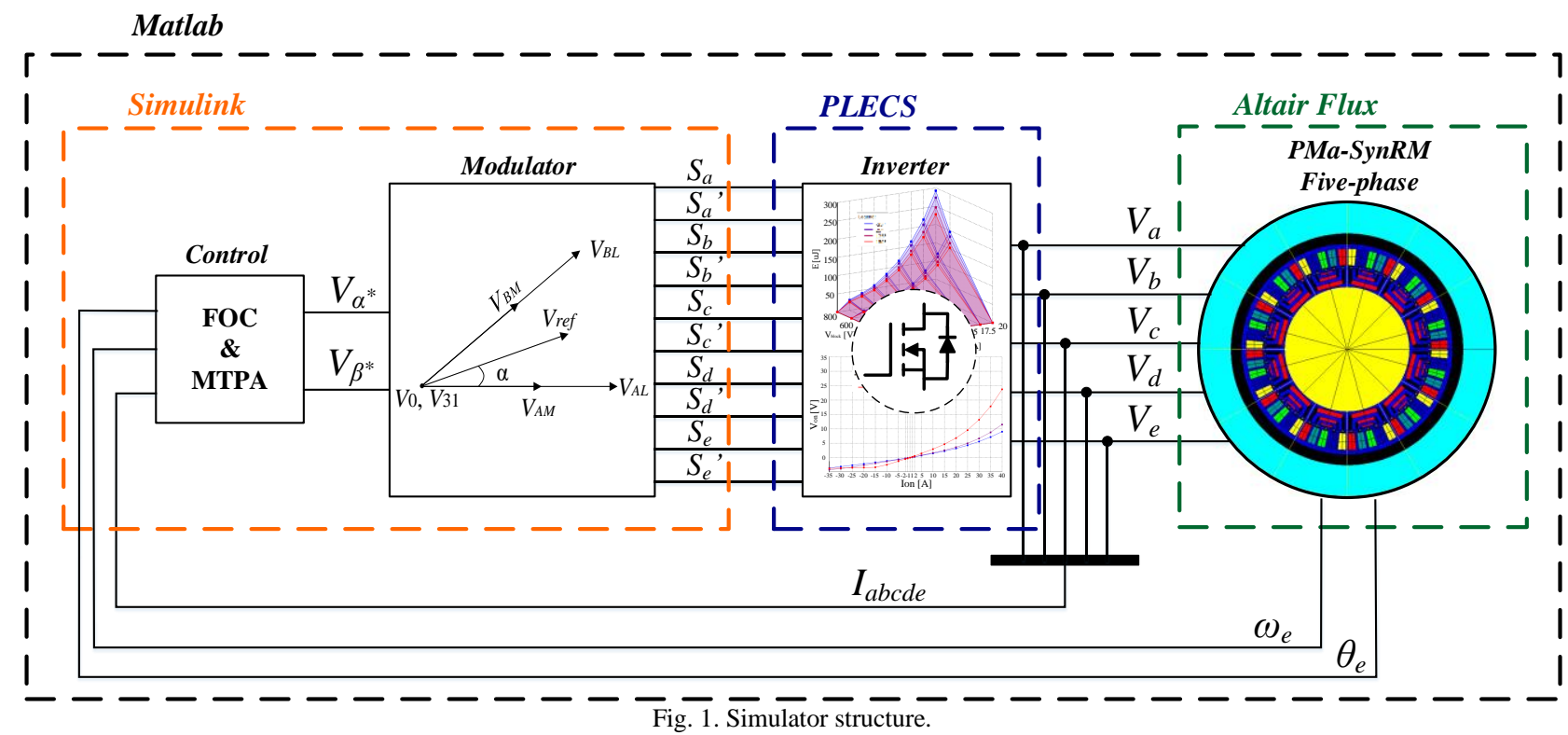

techniques for dividing a complex problem into small elements, which form a subdomain that is represented by a set of element equations for the original complex problem. FEA simulations are an important resource for removing multiple instances of creation and test of prototypes in various situations. The benefits of Altair Flux are the accuracy and reliability of its solvers, its flexibility in adapting to the user's specific needs, its ability to couple with other software for high productivity and to obtain the most realistic representation of a system. Altair Flux can be used in a wide variety of areas, for example on magnetic, electric and thermal fields, mechanical, multiphysics, magnetic, and thermal coupling, as well as others.

The main objective of this paper is to provide a tool that allows us to evaluate the performance of the electric motor design. In addition, it also allows you to evaluate control techniques, study the behavior of modulation techniques and their influence on the power inverter. All this is achieved through the use of a friendly user interface that allows us to quickly and easily configure the conditions under which the simulation will take place.

\section{THE SimulatoR}

The simulation platform consists of five parts: the control phase, the modulation block, the electronic inverter, the electrical machine, and the user interface. The control phase and modulation block are implemented in Simulink/MATLAB. The electronic inverter is designed in PLECS. And the five-phase Permanent Magnet assisted Synchronous Reluctance Motor (PMa-SynRM) is developed in Altair Flux. The user interface was made with a GUIDE in MATLAB.

\section{A. Control}

Field-Oriented Control (FOC) is the control technique applied in this software. Fig. 2 shows the diagram block of the FOC implemented in Simulink/MATLAB. This control consists of the two internal current loops and one external speed loop. An anti-windup (AWU) loop is added to saturate the amplitude of the reference voltage. The speed loop uses a Maximum Torque Per Ampere (MTPA) table to get the two currents signals for the internal loops [15], [16]. The MTPA was obtained from a previous study of PMa-SynRM conducted in Altair Flux. That study had the purpose of obtaining the current values needed to generate the maximum torque of the motor. Here we obtain variations in the values of the inductances and in the flow of the permanent magnets based on the currents needed to generate the maximum torque. Fig. 3 shows the MTPA curves, where the red line is the torque generated by the currents.

The FOC was designed with a block diagram in Simulink. Clark and Park direct and inverse transformations are needed for calculating FOC in order to convert the currents and voltages from the form abcde to $\alpha-\beta$ or to $d-q$ depending on the framework. This Clark-Park $\left(C P_{T}\right)$ direct transformation is applied as follows:

$$
C P_{T}=\left[\begin{array}{ccccc}
\cos (\theta) & \cos (\theta-2 \varphi) & \cos (\theta-4 \varphi) & \cos (\theta+4 \varphi) & \cos (\theta+2 \varphi) \\
-\sin (\theta) & -\sin (\theta-2 \varphi) & -\sin (\theta-4 \varphi) & -\sin (\theta+4 \varphi) & -\sin (\theta+2 \varphi)
\end{array}\right] \text { (1) }
$$

The feedback of the speed, the position of the rotor and the currents of the PMa-SynRM are obtained from the cosimulation model of Altair Flux. Also, the control must be adaptive, due to the changes in the inductances $L_{d}$ and $L_{q}$, and in the permanent magnet fluxes $\Phi_{P M d}$ and $\Phi_{P M q}$.

\section{B. Modulation block}

The modulation block allows selecting different types of modulation techniques and frequency switching for making the inverter will work.

The modulation techniques that can be used are the CBPWM and SVPWM. The CBPWM that is implemented is Sinusoidal PWM with the zero sequence injection.

For the SVPWM, the simulator has programmed three different types of modulations. These are the Two Large Vectors SVPWM (2L-SVPWM), Two Large Two Medium Vectors SVPWM and Four Large Vectors SVPWM. Each of them has one continuous mode and four discontinuous modes. 


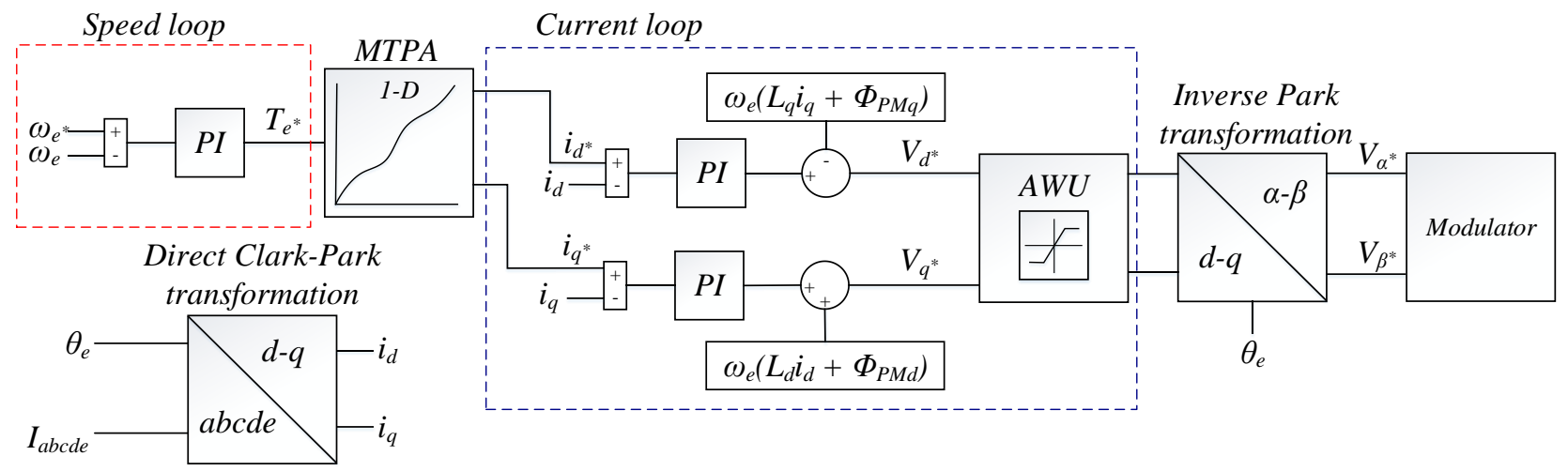

Fig. 2. FOC diagram block with MTPA.

This block receives the reference voltage in the frame $\alpha-\beta$ from the control. Depending on the selected modulation technique, if the CBPWM is applied, the block transforms the reference voltage from the frame $\alpha-\beta$ to abcde with a normalized magnitude (between -1 to 1 of amplitude) before injecting the zero sequence. If the selected technique is SVPWM the block calculates the duty cycle for activating the space vectors that are needed to reach the reference vector.

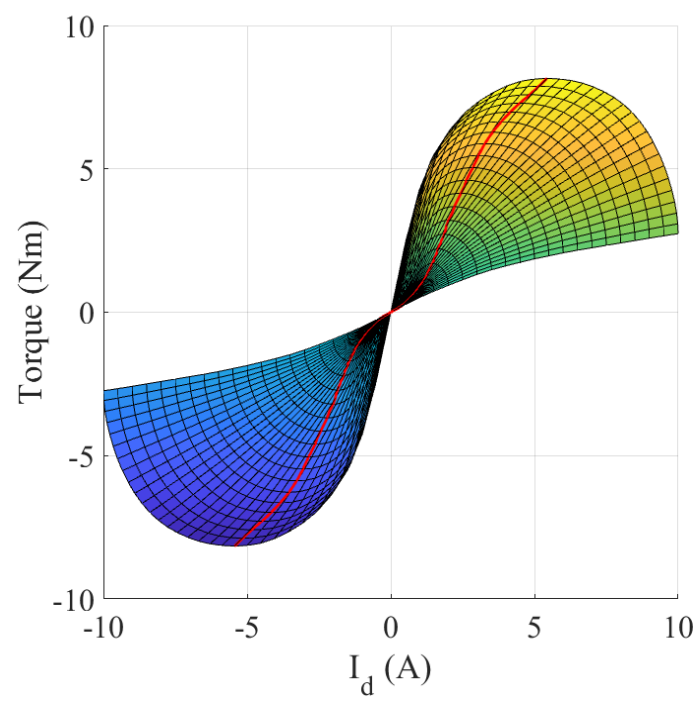

(a)

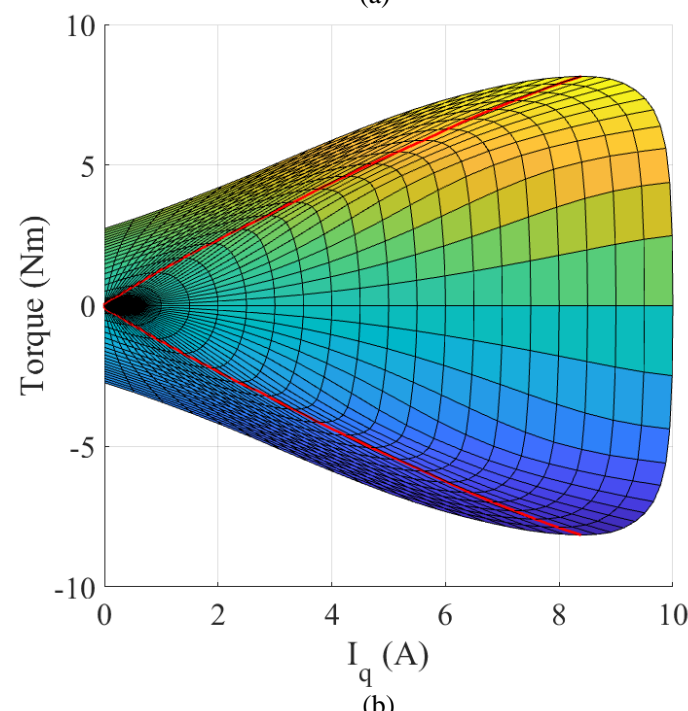

Fig. 3. MTPA Curves: (a) torque vs $I_{d}$ and (b) torque vs $I_{q}$.
The outputs of the block are the activation signals of the semiconductor devices in the power inverter.

\section{Five-Phase Inverter}

The simulation model of the five-phase VSI is designed in the PLECS blockset form Simulink/MATLAB.

PLECS allows to obtain the values of the switching and conduction losses for each semiconductor device. This is due to the use of lookup tables and formula that are directly obtained from the manufacturer of the semiconductor devices. PLECS has a heat shrink function that emulates the working temperature of the components inside the inverter, this help to calculate in a more accurate way the values of the semiconductors devices currents, voltages, and losses. With the losses we can be able to calculate the efficiency of the inverter.

\section{1) Conduction losses}

When MOSFET is in the on-state the conduction losses appear. These losses are defined as follows:

$$
P_{\text {condloss }}=\frac{1}{T_{s}} \int_{0}^{T_{s}}\left(V_{F 0}+R_{o n} I_{F}\right) * I_{F} d t
$$

Where $T_{s}$ is the period of the fundamental frequency, $V_{F 0}$ is the threshold voltage, $R_{o n}$ is the on resistance and $I_{F}$ is the forward current.

\section{2) Switching losses}

Switching losses are generated when the turn-on and turnoff switching processes occur in the power devices, and these defined in the form of:

$$
P_{\text {Swtloss }}=\frac{1}{T_{s}} \sum_{j=1}^{n T_{s}}\left[E_{\text {on }}\left(I_{F}, V_{\text {offs }}\right)+E_{\text {off }}\left(I_{F}, V_{\text {offs }}\right)\right]
$$

where $n T_{s}$ is the number of transitions in one fundamental period, $E_{\text {on }}$ and $E_{\text {off }}$ are the turn-on and turn-off energy dissipated during the change of state, $I_{F}$ is the on-state current running through the power devices and $V_{\text {offs }}$ is the voltage in the off-state.

\section{3) Efficiency}

Efficiency is defined in the following form: 


$$
\eta(\%)=\left(\frac{P_{\text {tot }}-P_{\text {condloss }}-P_{\text {swtloss }}}{P_{\text {tot }}}\right) * 100
$$

where $P_{\text {tot }}$ is the inverter total power.

Hence, PLECS calculates the conduction and switching losses based on the previous equations and the lookup tables for the semiconductor devices. For this case, Fig. 4 shows the lookup tables of the selected device, the MOSFET SiC C2M0160120D from the manufacturer CREE.

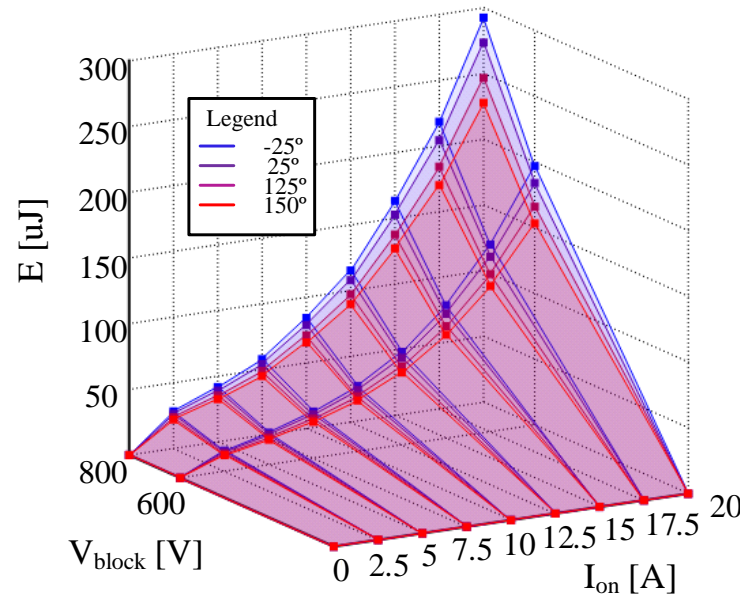

(a)

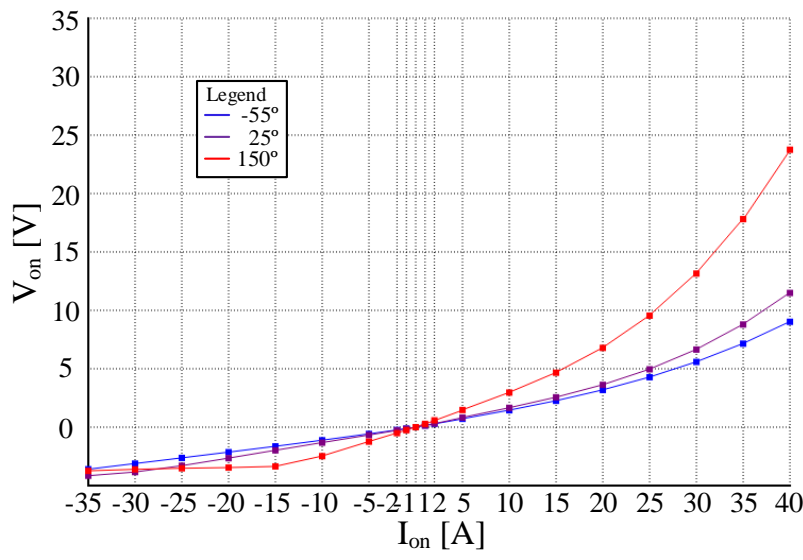

(b)

Fig. 4. PLECS lookup tables for the MOSFET C2M0160120D: (a) Switching and (b) conduction losses.

\section{Five-phase Permanent Magnet assisted Synchronous Reluctance Motor (PMa-SynRM)}

The Five-phase Permanent Magnet assisted Synchronous Reluctance Motor is implemented in the software Altair Flux. The 2-D model geometry and calculation mesh of the PMaSynRM are designed in Altair Flux. Also, is necessary to configure some values and characteristics, such as the number of spires of the coils, the density flux value of the permanent magnet, the magnetic properties of the used material in the motor, and various others. Fig. 5 shows the 2D design of the PMa-SynRM in Altair Flux.

Moreover, it is essential to design the electrical circuit of the PMa_SynRM so that the electrical machine perform similarly to real conditions.

The flexibility and ability to interconnect with other software, such as MATLAB is necessary for creating multiphysics inputs and outputs in Flux. In this case, where the simulation kinematics are coupled to a load, the inputs for the motor model are the five voltages abcde and the coupled load. The outputs will be: currents, motor torque and speed, and rotor position.

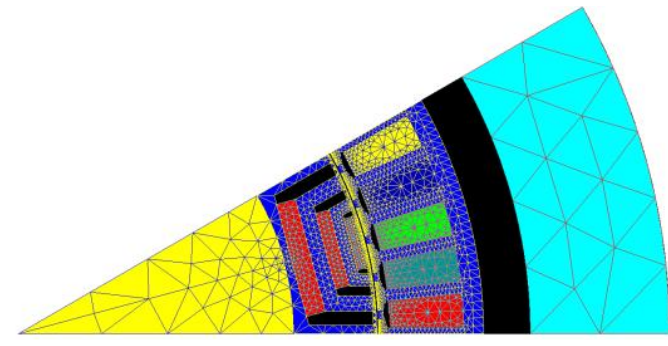

(b)

Fig. 5. Meshing of one pole of the PMa-SynRM.

\section{E. User Interface}

The user interface that was developed in a GUIDE on MATLAB and it allows to configure all the simulation parameters. This interface consists of three parts: parameter configuration, data area, and plot area.

The parameter configuration function allows selecting the modulation technique and the switching frequency. It can also configure the speed and torque references, working temperature, simulation cycles, and the harmonic content that can be observed in the voltage and currents signal.

It additionally has the buttons for starting and stopping the simulation.

The data area has the function of select the information to be displayed in the plots. The available information is: phaseto-neutral point voltage, phase-to-phase voltage, currents, switching and conduction losses, gate signal, joint temperature, $\mathrm{SiC}$ currents and voltages, and harmonic content for voltages and currents. The total switching and conduction losses as well as the simulation status can also be observed. The Export Data button allows selecting the data and exporting it to an excel file.

\section{SIMULATION}

Following some simulations are carried out with the develop platform. First, the modulation and the switching frequency are configured. Second, the speed and torque (load) references are set. Finally, the temperature of the heat sink is introduced in PLECS, the harmonic content that will be observed, and the simulation time expressed in fundamental frequency cycles. Table I shows the simulation parameters applied in this work. Table II shows the five-phase PMaSynRM; parameters.

TABLE I. SIMULATION PARAMETERS.

\begin{tabular}{|c|c|}
\hline Modulation technique & 2L SVPWM \\
\hline Switching frequency & $10 \mathrm{kHz}$ \\
\hline Speed reference & $1000 \mathrm{rpm}$ \\
\hline Torque reference & $4 \mathrm{Nm}$ \\
\hline Temperature & $150^{\circ} \mathrm{C}$ \\
\hline Simulation cycles & 200 \\
\hline Number of harmonics & 40 \\
\hline$d c$ bus link & $550 \mathrm{~V}$ \\
\hline
\end{tabular}

Once the simulation parameters have been set, we proceed to simulate and compare the results obtained with the simulator and a solely MATLAB-based model. The objective is to observe the behavior of both systems, and look at their similarities and differences. 
TABLE II. PMA_SYNRM PARAMETERS.

\begin{tabular}{|c|c|}
\hline Nominal Power & $3 \mathrm{~kW}$ \\
\hline Nominal Torque & $7 \mathrm{Nm}$ \\
\hline Nominal phase current & $4 \mathrm{~A} \mathrm{rms}$ \\
\hline Stator resistance & $2.5 \Omega$ \\
\hline Nominal speed & $4000 \mathrm{rpm}$ \\
\hline Number of phases & 5 \\
\hline Pair of poles & 6 \\
\hline
\end{tabular}

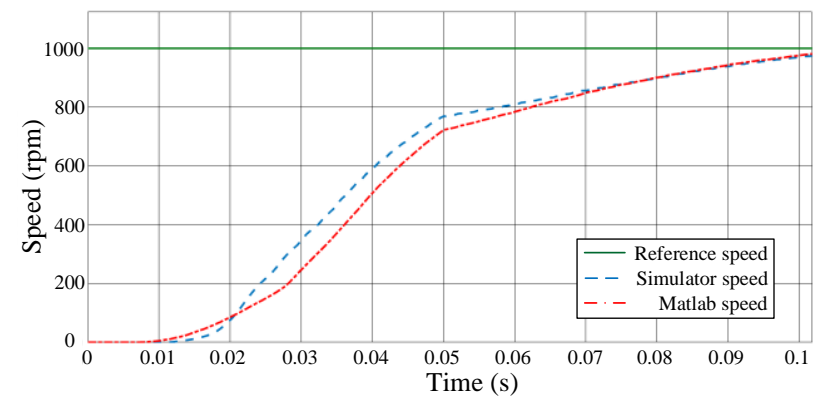

Fig. 6. Speed response

The results obtained show that both systems respond in a similar way. On the one hand, the simulator results are more precise due to the fact that PLECS and Altair Flux are used in order to characterize the inverter and the motor models respectively. On the other hand, the MATLAB-based model simulates $95 \%$ faster than the simulator.

Fig. 7 shows the torque response of both models. It can be observed that the initial response in both models is not the same. The simulator response (blue line) shows a higher toque peak than the MATLAB-based model. Furthermore, when torque values close to the reference are reached, the amplitude of the oscillations obtained with the simulator are still higher.

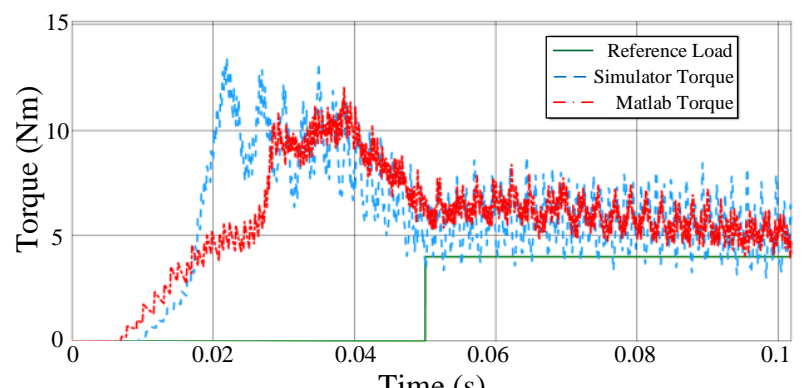

Fig. 7. Torque response comparison between simulator and MATLABbased model.

In Fig. 8 and 9 the currents and their harmonic content are displayed. The currents obtained by the simulator present more harmonic content than the ones obtained with the MATLAB-based model. This is because the MATLAB-based model has been elaborated considering only the first harmonic electromagnetic flows.

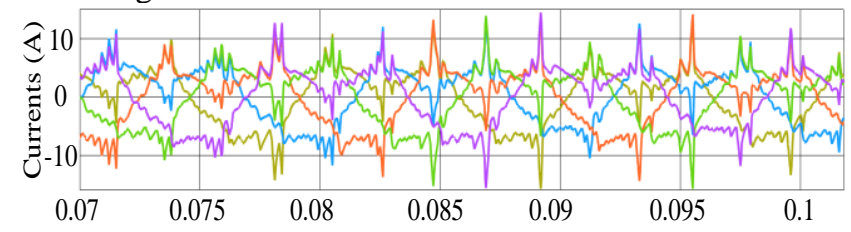

(a)

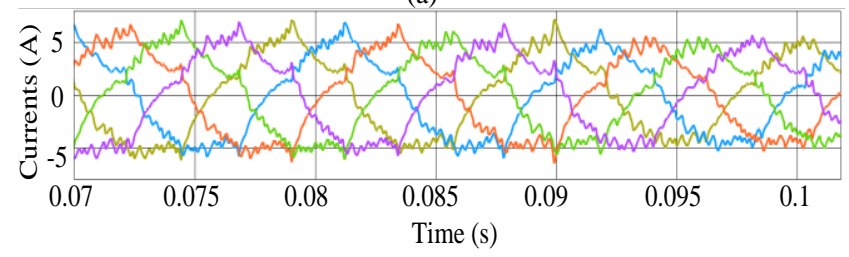

(b)

Fig. 8. Currents: (a) simulator, and (b) MATLAB-based model.

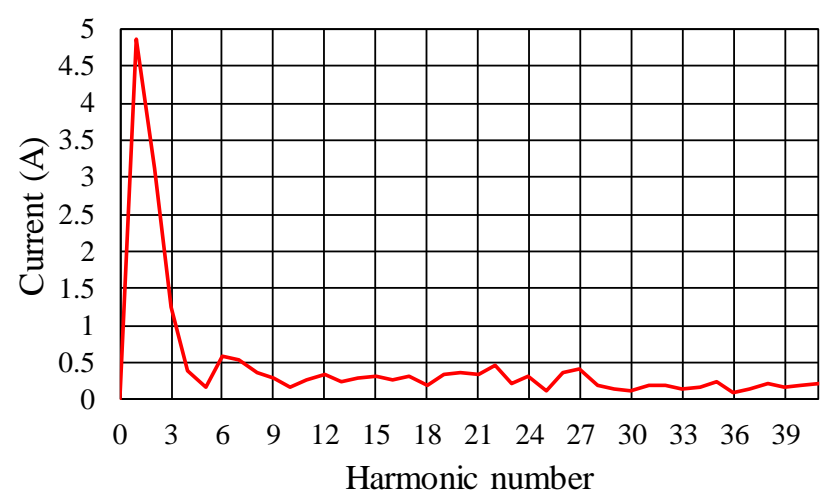

(a)

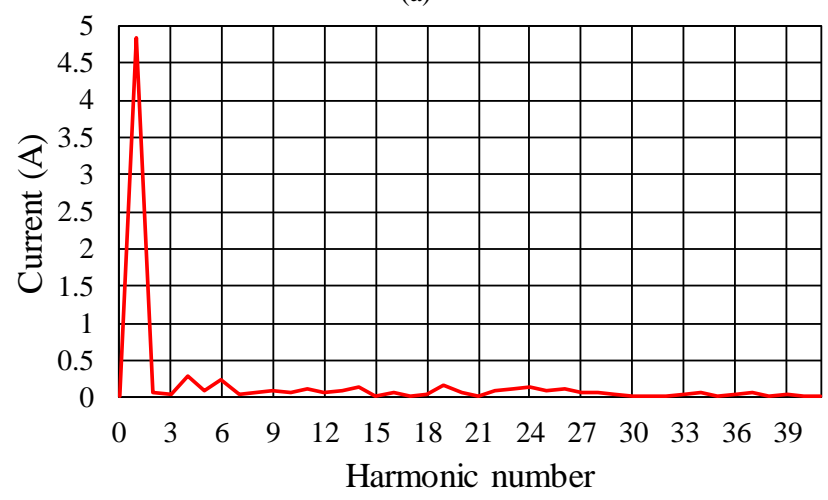

(b)

Fig. 9. Current harmonic content: (a) simulator, and (b) MATLAB-base model.

Fig 10 shows the inverter efficiency of both models. MATLAB-based model inverter shows a better efficiency than the simulator platform inverter. This is due to the better accuracy of the results obtained with PLECS and Altair flux in comparison with the ones of the MATLAB-based model.

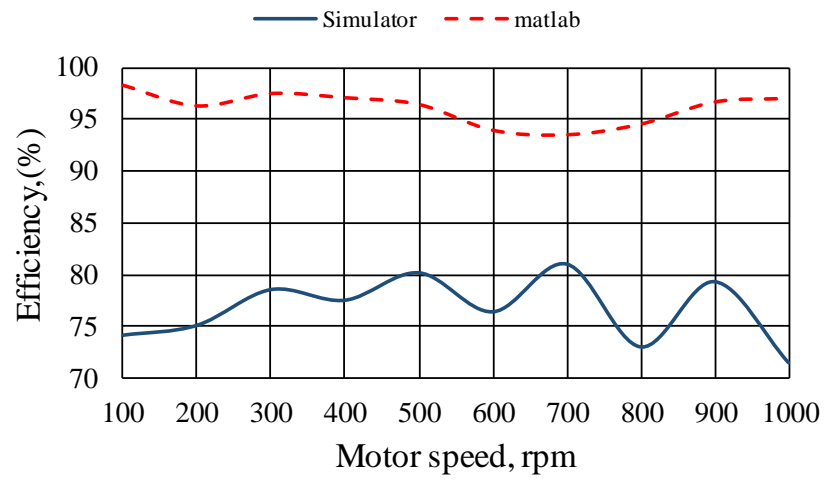

Fig. 10. Efficiency calculation comparison between the simulator and the MATLAB-based model.

\section{CONCLUSIONS}

Using a simulator helps us reduce the cost of designing and building an electromechanical system, but accurate results are mandatory. FEA-Altair Flux provides a more complete understanding of the motor's behavior. One disadvantage in obtaining these results is that the simulation time increases.

PLECS allows observing inverter performance based on semiconductor devices. These devices operate under certain conditions of temperature and modulation. While PLECS 
shows the inverter switching and conduction losses, simulation time increases as a result of calculating the losses.

The user interface helps make the simulator more userfriendly. This is because all the simulation parameters can be set up without the need to modify any of the main structures, making it easy and quick to configure the simulation.

Future works to improve the simulator will include selecting other control techniques, allowing changes in the inverter structure and selecting between various electrical machine.

Unlike other simulation platforms that use hardware in the loop or evaluate a defined traction system, this simulation platform will allow us to vary the elements of the traction system in an easy and simple way. With this we get versatility during design tests.

\section{ACKNOWLEDGMENT}

This work was supported by the Ministerio de Ciencia, Innovación y Universidades under Project CICYT (TRA2016-80472-R), the Consejo Nacional de Ciencia y Tecnología (CONACYT) of México (scholarship number 496458) and Secretaria d'Universitats i Recerca del Departament d'Empresa i Coneixement de la Generalitat de Catalunya.

\section{REFERENCES}

[1] D. Maksimovic, A. M. Stankovic, V. J. Thottuvelil, and G. C Verghese, "Modeling and simulation of power electronic converters," Proc. IEEE, vol. 89, no. 6, pp. 898-912, Jun. 2001

[2] M. Shixia, W. Hongfu, H. Junxian, and W. Tiezhu, "Fast Electromagnetic Transient Simulation Model of Photovoltaic Power System," in 2018 International Conference on Power System Technology (POWERCON), 2018.

[3] S. Doolla, S. S. Bhat, T. S. Bhatti, and M. Veerachary, "A GUI based simulation of power electronic converters and reactive power compensators using MATLAB/SIMULINK," in 2004 International Conference on Power System Technology, 2004. PowerCon 2004., 2005, vol. 2, no. November, pp. 1710-1715.

[4] K. K. Tse, H. S.-H. Hung, and S. Y. Hui, "Quadratic state-space modeling technique for analysis and simulation of power electronic converters," IEEE Trans. Power Electron., vol. 14, no. 6, pp. 1086$1100,1999$.
[5] S. George, R. V. Chacko, and A. Mathew, "Off-line and real-time simulation Modelling of Electric Vehicle power train," in 2014 Annual International Conference on Emerging Research Areas: Magnetics, Machines and Drives (AICERA/iCMMD), 2014, pp. 1-8

[6] O. Vodyakho, M. Steurer, C. S. Edrington, and F. Fleming, "An Induction Machine Emulator for High-Power Applications Utilizing Advanced Simulation Tools With Graphical User Interfaces," IEEE Trans. Energy Convers., vol. 27, no. 1, pp. 160-172, Mar. 2012.

[7] I. Lopez et al., "Generalized PWM-Based Method for Multiphase Neutral-Point-Clamped Converters With Capacitor Voltage Balance Capability," IEEE Trans. Power Electron., vol. 32, no. 6, pp. 48784890, Jun. 2017

[8] F. Barrero and M. J. Duran, "Recent Advances in the Design, Modeling, and Control of Multiphase Machines-Part I," IEEE Trans. Ind. Electron., vol. 63, no. 1, pp. 449-458, Jan. 2016.

[9] E. Levi, "Multiphase Electric Machines for Variable-Speed Applications," IEEE Trans. Ind. Electron., vol. 55, no. 5, pp. $1893-$ 1909, May 2008.

[10] J. Prieto, F. Barrero, S. Toral, E. Levi, M. Jones, and M. J. Durán, "Analytical Evaluation of Switching Characteristics in Five-Phase Drives with Discontinuous Space Vector Pulse Width Modulation Techniques,” EPE J., vol. 23, no. 2, pp. 24-33, Jun. 2013.

[11] W. N. Wan Abdul Munim, M. F. Ismail, A. F. Abidin, and H. Che Mat Haris, "Switching technique comparison for multi-phase inverters," in 2013 IEEE 7th International Power Engineering and Optimization Conference (PEOCO), 2013, no. June, pp. 155-160.

[12] S. C. Rangari, "Implementation of Large and Medium Vectors for SVPWM technique in five phase Voltage Source Inverter," in International Conference on Intelligent Computing and Control Systems (ICICCS), 2017, no. 1, pp. 751-756.

[13] J. Prieto, M. Jones, F. Barrero, E. Levi, and S. Toral, "Comparative Analysis of Discontinuous and Continuous PWM Techniques in VSIFed Five-Phase Induction Motor," IEEE Trans. Ind. Electron., vol. 58, no. 12 , pp. $5324-5335$, Dec. 2011

[14] E. Gurpinar and A. Castellazzi, "Single-Phase T-Type Inverter Performance Benchmark Using Si IGBTs, SiC MOSFETs and GaN HEMTs," IEEE Trans. Power Electron., vol. 31, no. 10, pp. 1-1, 2015.

[15] S. Wang, M. Degano, J. Kang, A. Galassini, and C. Gerada, "A Novel Newton-Raphson-Based Searching Method for the MTPA Control of Pmasynrm Considering Magnetic and Cross Saturation," in 2018 XIII International Conference on Electrical Machines (ICEM), 2018, pp. 1360-1366.

[16] R. Thike and P. Pillay, "Experimental Investigation of MTPA Trajectory of Synchronous Reluctance Machine," in 2018 IEEE International Conference on Power Electronics, Drives and Energy Systems (PEDES), 2018, pp. 1-6. 